\title{
Häufig gebrauchte Symbole
}

\begin{tabular}{|c|c|c|c|}
\hline$A$ & Madelung-Faktor & $\Delta G$ & freie Reaktionsenthalpie \\
\hline$A_{\mathrm{r}}$ & relative Atommasse & $\Delta G^{\neq}$ & freie Aktivierungsenthalpie \\
\hline \multirow[t]{3}{*}{$a$} & Aktivität & $g$ & gerade \\
\hline & Ionenladungszahl & & (als Index) gasförmig \\
\hline & Strecke & & Metall-Faktor d. spektrochemi- \\
\hline$a_{\mathrm{o}}$ & Bohrscher Radius & & schen Reihe \\
\hline B & Base & & - Faktor der Elektronen \\
\hline \multirow[t]{2}{*}{$B$} & $\begin{array}{l}\text { Faktor d. Abstoßungsenergie n. } \\
\text { Born }\end{array}$ & $\Delta H$ & $\begin{array}{l}\text { Enthalpie } \\
\quad \text { Atomisierungs- } \Delta H_{\mathrm{A}}\end{array}$ \\
\hline & $\begin{array}{l}\text { Racah-Parameter f. freies Metall- } \\
\text { Ion }\end{array}$ & & $\begin{array}{ll}\text { Bildungs- } & \Delta H_{\mathrm{B}} \\
\text { Reaktions- } & \Delta H_{\mathrm{R}}\end{array}$ \\
\hline$B^{\prime}$ & $\begin{array}{l}\text { Racah-Parameter f. komplexiertes } \\
\text { Metall-Ion }\end{array}$ & $h$ & $\begin{array}{l}\text { nephelauxetischer Parameter für } \\
\text { Liganden }\end{array}$ \\
\hline$b$ & Ladungskoeffizient & & Planck-Konstante (,Wirkungs- \\
\hline \multirow[t]{2}{*}{$b$} & Ionenladungszahl & & quantum") \\
\hline & Strecke & $\hbar$ & $h / 2 \pi$ \\
\hline \multirow[t]{3}{*}{$C$} & $\begin{array}{cc}\text { Kovalenz-Parameter } \\
\text { Base- } & C_{\mathrm{B}} \\
\text { Säure- } & C_{\mathrm{S}}\end{array}$ & $J$ & $\begin{array}{l}\text { NMR-Kopplungskonstante } \\
\text { Gesamtdrehimpulsquantenzahl } \\
\quad(L+S)\end{array}$ \\
\hline & Racah-Parameter f. freies Metall- & $j$ & Quantenzahl $(l+s)$ \\
\hline & $\begin{array}{l}\text { Iö } \\
\text { Wärmekapazität }\end{array}$ & $\begin{array}{l}\mathrm{K} \\
K\end{array}$ & $\begin{array}{l}\text { Elektronenschale mit } n=1 \\
\text { Gleichgewichtskonstante }\end{array}$ \\
\hline$C^{\prime}$ & $\begin{array}{l}\text { Racah-Parameter f. komplexiertes } \\
\text { Metall-Ion }\end{array}$ & & $\begin{array}{ll}\text { Basereaktionen } & K_{\mathrm{B}} \\
\text { Reaktionen i. Lsg. } & K_{c}\end{array}$ \\
\hline \multirow[t]{2}{*}{$c$} & $\begin{array}{l}\text { Konzentration } \\
\text { Lichtgeschwindigkeit }\end{array}$ & & $\begin{array}{ll}\text { Gasreaktionen } & K_{p} \\
\text { Säurereaktionen } & K_{\mathrm{S}}\end{array}$ \\
\hline & Strecke & $K_{\mathrm{AB}}$ & Ionenprodukt d. Verbindung $\mathrm{AB}$ \\
\hline $\mathrm{D}$ & $\begin{array}{l}\text { Debye (alte Einheit } \mathrm{f} \text {. Dipol- } \\
\text { moment) }\end{array}$ & $k$ & $\begin{array}{l}\text { Konstante } \\
\text { nephelauxetischer Parameter für }\end{array}$ \\
\hline D & Konfigurationskennzeichnung & & Metall-Ionen \\
\hline$D$ & $\begin{array}{l}\text { Atomzustand; Termsymbol } \\
\quad(L=2)\end{array}$ & $\begin{array}{l}\mathrm{L} \\
\mathrm{L}\end{array}$ & $\begin{array}{l}\text { Elektronenschale mit } n=2 \\
\text { Konfigurationskennzeichnung }\end{array}$ \\
\hline$d$ & $\begin{array}{l}\text { Abstand, Durchmesser } \\
\text { Atomorbital }(l=2) \\
\text { rechtsdrehend }\end{array}$ & $L$ & $\begin{array}{l}\text { austretende (leaving) Gruppe } \\
\text { Quantenzahl f. Mehrelektronen- } \\
\text { atom }\end{array}$ \\
\hline \multirow[t]{3}{*}{$E$} & $\begin{array}{l}\text { eintretende (entering) Gruppe } \\
\text { elektrostatischer Parameter }\end{array}$ & $l$ & $\begin{array}{l}\text { Länge } \\
\text { linksdrehend }\end{array}$ \\
\hline & $\begin{array}{ll}\text { Base- } & E_{\mathrm{B}} \\
\text { Säure- } & E_{\mathrm{S}}\end{array}$ & & $\begin{array}{l}\text { Quantenzahl (Bahndrehimpuls-, } \\
\text { Neben-) }\end{array}$ \\
\hline & Energie & $M$ & (Spin-)Multiplizität \\
\hline & Basis des natürlichen Logarithmus & $M_{\mathrm{r}}$ & relative Molekülmasse \\
\hline & Elementarladung & $m$ & (Elektronen-)Masse \\
\hline$e^{-}$ & Elektron & & Molalität \\
\hline \multirow[t]{3}{*}{$F$} & $\begin{array}{l}\text { Atomzustand; Termsymbol } \\
(L=3)\end{array}$ & & $\begin{array}{l}\text { Anzahl } \\
\text { magnetische Quantenzahl }\end{array}$ \\
\hline & Faraday-Konstante & $\begin{array}{l}m_{l} \\
m_{s}\end{array}$ & magnetische Spinquantenzahl \\
\hline & Farbzentrum ( $F$-Zentrum) & $N$ & Normierungsfaktor \\
\hline \multirow[t]{4}{*}{$f$} & Atomorbital $(l=3)$ & & Zahl \\
\hline & Feldfaktor $\mathrm{f}$. Liganden & $N_{\mathrm{A}}$ & Avogadro-Zahl \\
\hline & (als Index) fest & $n$ & Anzahl \\
\hline & Verteilungsfaktor & & Born-Exponent \\
\hline
\end{tabular}


XXX Häufig gebrauchte Symbole

\begin{tabular}{|c|c|c|c|}
\hline \multirow[t]{2}{*}{$n$} & Brechungsindex & Zeichen & $\simeq$ etwa gleich \\
\hline & Hauptquantenzahl & & $\hat{=}$ entsprechend \\
\hline \multirow[t]{3}{*}{$P$} & $\begin{array}{l}\text { Atomzustand; Termsymbol } \\
\quad(L=1)\end{array}$ & & $\begin{array}{l}(+),(-) \text { formale Ladungen } \\
\equiv \text { identisch }\end{array}$ \\
\hline & Paarbildungsenergie & & $\sim$ proportional \\
\hline & $\begin{array}{l}\text { prozentualer } p \text {-Charakter eines } \\
\text { Orbitals }\end{array}$ & & $\approx$ ungefähr \\
\hline p & $\begin{array}{l}\text { negativer dekadischer Loga- } \\
\text { rithmus }\end{array}$ & $\alpha$ & Kraftkonstante d. Streckschwin- \\
\hline$p$ & Atomorbital $(l=1)$ & & \\
\hline$Q$ & Kernquadrupolmoment & $\lceil\alpha\rceil$ & $\begin{array}{l}\text { Polarisierbarkeit } \\
\text { spezifische Drehung }\end{array}$ \\
\hline$q$ & $\begin{array}{l}\text { elektrischer Feldgradient } \\
\text { Ladung }\end{array}$ & $\beta$ & Kraftkonstante d. Knickschwin- \\
\hline $\mathrm{R}$ & Konfigurationsbezeichnung & & MOSE-Konstante \\
\hline \multirow[t]{2}{*}{$R$} & Güte-Faktor & & nephelauxetisches Verhältnis $\frac{B}{D}$ \\
\hline & $\begin{array}{l}\text { Radiale Wellenfunktion } \\
\text { allgemeine Gaskonstante }\end{array}$ & $\Delta$ & Differenz $\quad B$ \\
\hline \multirow[t]{2}{*}{$r$} & Polarkoordinate & & Enantiomerenkennzeichnung \\
\hline & Radius & & Energieaufspaltung im Liganden- \\
\hline \multirow[t]{2}{*}{$\mathrm{S}$} & Konfigurationsbezeichnung & & feld $(\equiv 10 D q)$ \\
\hline & Säure & & Wärmeenergie \\
\hline \multirow[t]{7}{*}{$S$} & Abschirmungskonstante & $\delta$ & chemische Verschiebung \\
\hline & $\begin{array}{l}\text { Atomzustand; Termsymbol } \\
\quad(L=0)\end{array}$ & & $\begin{array}{l}\text { Konformationskennzeichnung } \\
\text { Molekülorbital }\end{array}$ \\
\hline & Gesamtspin $\left(\Sigma_{S}\right)$ & & Partialladung \\
\hline & prozentualer $s$-Charakter eines & $\delta_{1}$ & Aufspaltung des $e_{g}$-Niveaus \\
\hline & Orbitals & $\delta_{2}$ & Aufspaltung des $t_{2 \mathrm{~g}}$-Niveaus \\
\hline & $\begin{array}{l}\text { Quantenzahl f. Mehrelektronen- } \\
\text { atom }\end{array}$ & $\varepsilon$ & $\begin{array}{l}\text { Dielektrizitätskonstante } \\
\text { molarer Extinktionskoeffizient }\end{array}$ \\
\hline & Überlappungsintegral & & Substituenteneinflußfaktor \\
\hline \multirow{3}{*}{$\Delta S$} & Entropie & $\varepsilon_{\mathrm{o}}$ & elektrische Feldkonstante (Dielek- \\
\hline & Atomorbital $(l=0)$ & & trizitätskonstante d. Vakuums) \\
\hline & Elektronenspin & $\mathscr{E}$ & elektromotorische Kraft \\
\hline \multirow[t]{2}{*}{$T$} & absolute Temperatur & $\eta$ & hapto \\
\hline & trans-dirigierende Gruppe & $\dot{\Theta}$ & $\vartheta$-abhängige Wellenfunktion \\
\hline \multirow[t]{2}{*}{$t$} & transfer-Parameter & $\vartheta$ & Polarkoordinate \\
\hline & $\begin{array}{ll}\text { Base- } & t_{\mathrm{B}} \\
\text { Säure- } & t_{\mathrm{S}}\end{array}$ & $\Lambda$ & $\begin{array}{l}\text { Enantiomerenkennzeichnung } \\
\text { Konformationskennzeichnung }\end{array}$ \\
\hline$U$ & Gitterenergie & $\lambda$ & $\begin{array}{l}\text { Wellenlänge } \\
\text { Welchnung }\end{array}$ \\
\hline$u$ & atomare Masseeinheit & $\mu$ & Dipolmoment \\
\hline$u$ & ungerade & & magnetisches Moment \\
\hline \multirow[t]{4}{*}{$V$} & Potential & $\mu$ - & Brücke(n-Ligand) \\
\hline & potentielle Energie & $v$ & Frequenz \\
\hline & Valenzzustand & & Teilchenzahl \\
\hline & Volumen & $\tilde{v}$ & Wellenzahl $(=1 / \lambda)$ \\
\hline$\Delta V^{*}$ & Aktivierungsvolumen & $\pi$ & Molekülorbital \\
\hline \multirow[t]{2}{*}{$x$} & Molenbruch & $\sum$ & Summe \\
\hline & Raumkoordinate & $\bar{\sigma}$ & Molekülorbital \\
\hline & Raumkoordinate & $\Phi$ & $\varphi$-abhängige Wellenfunktion \\
\hline$Z$ & $\begin{array}{l}\text { Kernladungszahl, Ordnungszahl } \\
\text { (Atomnummer) }\end{array}$ & $\begin{array}{l}\phi \\
{[\phi]}\end{array}$ & $\begin{array}{l}\text { Ionenpotential } \\
\text { molekulare Drehung }\end{array}$ \\
\hline \multirow{3}{*}{$\begin{array}{l}Z_{\text {eff }} \\
z\end{array}$} & effektive Kernladungszahl & $\varphi$ & Polarkoordinate \\
\hline & Ionenladungszahl & $\chi$ & Elektronegativität \\
\hline & Raumkoordinate & $\Psi$ & Wellenfunktion \\
\hline
\end{tabular}

